\title{
Oxaliplatin-induced hepatic sinusoidal obstruction syndrome in a patient with gastric cancer: A case report
}

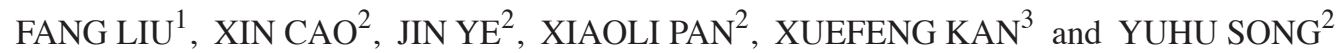 \\ ${ }^{1}$ Institute of Hematology, ${ }^{2}$ Division of Gastroenterology, and ${ }^{3}$ Department of Radiology, Union Hospital, \\ Tongji Medical College, Huazhong University of Science and Technology, Wuhan, Hubei 430022, P.R. China
}

Received April 29, 2017; Accepted December 14, 2017

DOI: $10.3892 / \mathrm{mco} .2017 .1540$

\begin{abstract}
Oxaliplatin is a platinum-based antineoplastic agent used in cancer chemotherapy. Oxaliplatin-induced hepatic sinusoidal obstruction syndrome (HSOS) has been reported in the context of chemotherapy for liver metastasis of colorectal cancer. However, to the best of our knowledge, there is no report of oxaliplatin-associated HSOS in patients with gastric cancer. The present study reported a patient with gastric cancer who received proximal gastrectomy and oxaliplatin-containing chemotherapy. Contrast-enhanced computed tomography revealed that liver parenchyma appeared heterogeneous and demonstrated hypoattenuation in the portal phase. In dynamic contrast-enhanced magnetic resonance imaging, hypointense heterogeneity was demonstrated in the portal-venous phase. Pathological examination indicated distinctive multifocal sinusoidal dilatation. In conclusion, the present report indicated a case of oxaliplatin-induced HSOS in a patient with gastric cancer who received oxaliplatin-contained chemotherapy.
\end{abstract}

\section{Introduction}

Gastric cancer is one of the leading causes of cancer-related mortality worldwide. Despite a decrease in its incidence in some regions of the world, gastric cancer remains a major clinical challenge as the majority of cases are diagnosed in an advanced stage, with a poor prognosis and limited treatment options (1). Oxaliplatin, a platinum-based antineoplastic agent, is used as an essential part of chemotherapeutic regimens for colorectal liver metastasis (2). Oxaliplatin-based regimens

Correspondence to: Dr Yuhu Song, Division of Gastroenterology, Union Hospital, Tongji Medical College, Huazhong University of Science and Technology, 1277 Jiefang Avenue, Wuhan, Hubei 430022, P.R. China

E-mail: yuhusong@yahoo.com

Abbreviations: HSOS, hepatic sinusoidal obstruction syndrome; CT, computed tomography; MRI, magnetic resonance imaging

Key words: oxaliplatin, sinusoidal obstruction syndrome, hepatic sinusoidal obstruction syndrome have been associated with the development of injury to the hepatic parenchyma in the form of hepatic sinusoidal obstruction syndrome (HSOS) (3-6). Since oxaliplatin-induced severe hepatic sinusoidal obstruction was first reported in 2004 (7), oxaliplatin-induced hepatic injury has become a major concern in patients with metastatic colorectal cancer (8-11). To the best of our knowledge, there have been no previously published reports on oxaliplatin-induced HSOS in other types of solid cancer. The present study described a case of oxaliplatin-induced HSOS in a patient with gastric cancer who received oxaliplatin-containing chemotherapy.

\section{Case report}

A 52-year-old man received gastroscopy because of dysphagia at Union hospital, Tongji Medical College (Wuhan, China) in Feb 2016. Gastroscopic examination indicated cardia-fundus neoplasia and pathological examination demonstrated gastric adnenocarcinoma. Subsequently, the patient underwent proximal gastrectomy for cardia-fundus gastric carcinoma and the pathological diagnosis was stage pT3N0M0 moderately and well-differentiated tubular adenocarcinoma. For treatment, the patient received five cycles of $\mathrm{S}-1$ and oxaliplatin (SOX) regimen, consisting of intravenous injection of $130 \mathrm{mg} / \mathrm{m}^{2}$ oxaliplatin (Sanofi Pharmaceutical, Gentilly, France) on day 1 followed by oral administration of $50 \mathrm{mg}$ TGOP capsules (50 mg tegafur, $14.5 \mathrm{mg}$ gimeracil and $49 \mathrm{mg}$ oteracil potassium; Qilu Pharmaceutical Co., Ltd., Jinan, China) twice daily on days $1-14$, every 3 weeks. Following the five cycles of SOX, the patient presented no abnormal symptoms, and the results of blood routine examination and liver function tests were normal. The patient had no previous history of liver disease and denied alcohol use.

The patient received a general check-up before the sixth cycle of SOX on schedule. Peripheral blood routine examination results were as follows: White blood cells, $3.79 \times 10^{9}$ cells/l; red blood cells, $3.38 \times 10^{12}$ cells/1; and platelet count, $179 \times 10^{9}$ cells $/ 1$. Liver function test results were as follows: Total bilirubin, $6.6 \mathrm{mmol} / 1$; direct bilirubin, $4.4 \mathrm{mmol} / 1$; alanine aminotransferase, $34 \mathrm{U} / 1$ (normal range, 21-72 U/1); aspartate aminotransferase, $55 \mathrm{U} / 1$ (normal range, 17-59 U/1); alkaline phosphatase, $190 \mathrm{U} / 1$ (normal range, 40-150 U/l); $\gamma$-glutamyl transferase, $58 \mathrm{U} / 1$ (normal range, 17-53 U/1); total protein, $64.9 \mathrm{~g} / \mathrm{l}$; and albumin, $32.1 \mathrm{~g} / \mathrm{l}$. Non-invasive fibrosis scores 

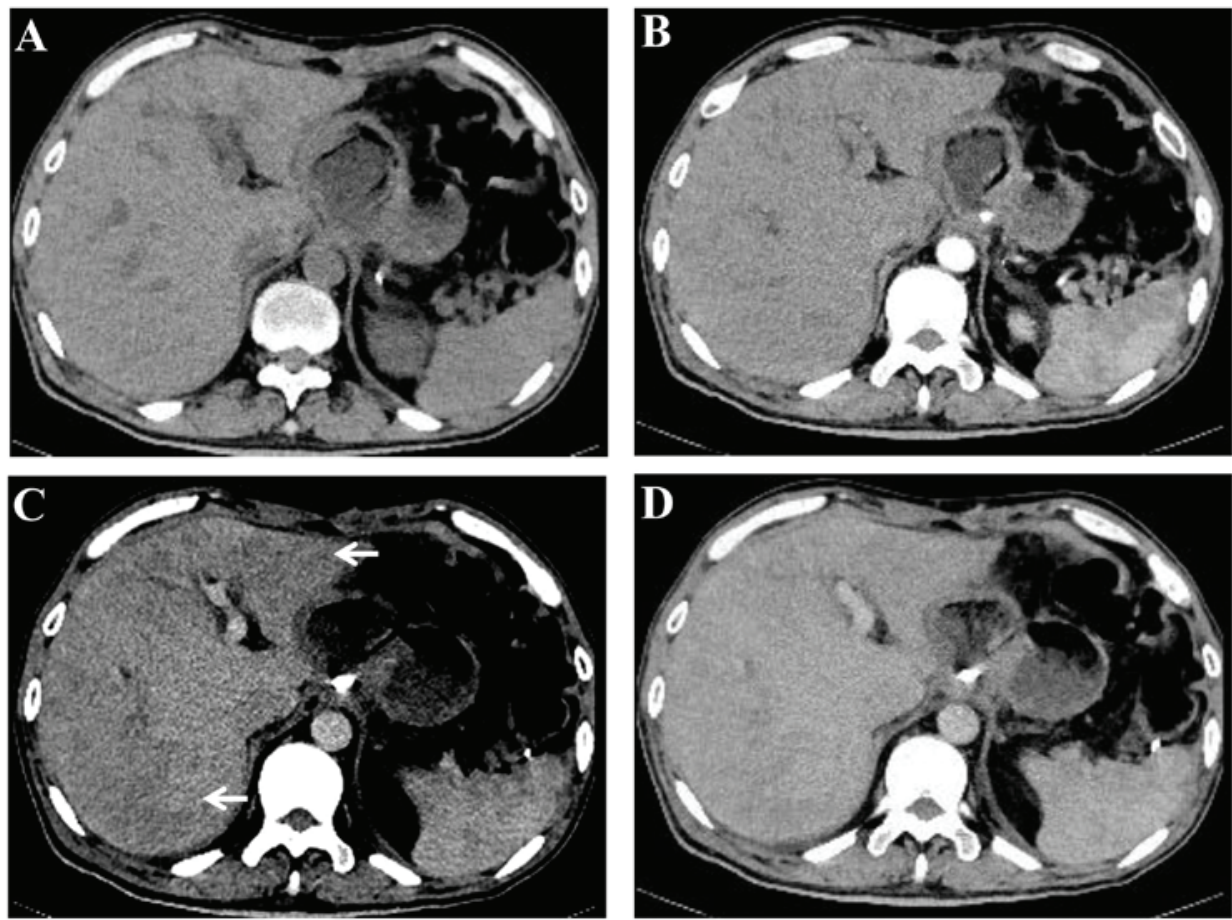

Figure 1. Contrast-enhanced CT demonstrating heterogeneous hypoattenuation in the liver. (A) Pre-contrast, (B) artery phase, (C) portal phase and (D) hepatic venous phase images. Heterogeneous hypoattenuation (arrows) was demonstrated in the portal phase of contrast CT. CT, computed tomography.
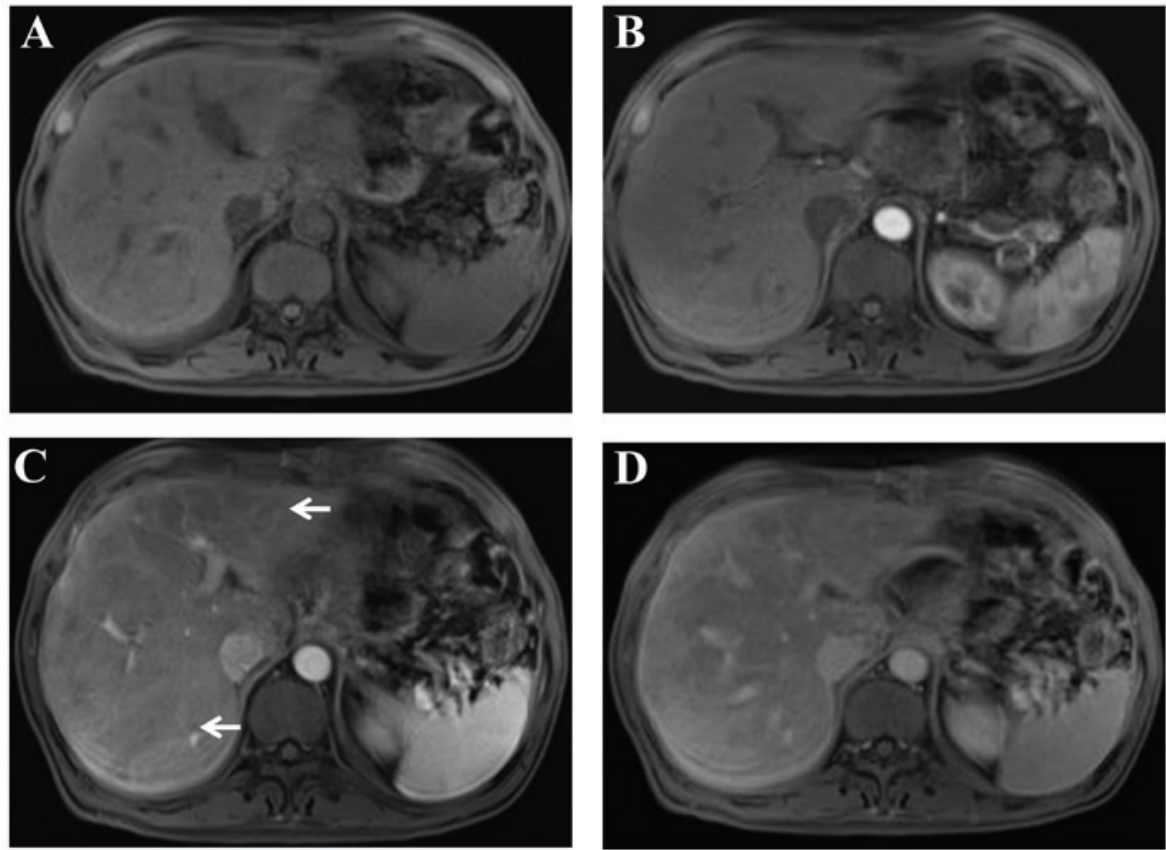

Figure 2. Liver parenchyma with a heterogeneous and predominantly hypointense appearance on T1-weighted gadolinium-enhanced MRI. (A) Pre-contrast, (B) artery phase, (C) portal phase and (D) hepatic venous phase images. A heterogeneous hypointense appearance (arrows) was revealed on the portal phase of contrast-enhanced MRI. MRI, magnetic resonance imaging.

were as follows: Aspartate aminotransferase to platelet ratio index, 0.52; and fibrosis-4 score, 2.74. Tests for hepatitis A, B, C, and E, Epstein-Barr virus, and cytomegalovirus infection were negative. Anti-nuclear antibody was weak positive; other autoantibodies were negative; immunoglobulin $\mathrm{G}$ level (18.3 g/l; normal range, 7.51-15.6 g/l) was increased and other immunoglobulin levels were within the normal range.
Enlargement of the lymph nodes was not observed in the patient through physical examination and ultrasonography. Contrast-enhanced computed tomography (CT) revealed that liver parenchyma appeared heterogeneous and demonstrated predominantly moderate hypoattenuation in the left and right lobe of the liver (Fig. 1) (8). On dynamic contrast-enhanced magnetic resonance imaging (12), heterogeneous hypointensity 


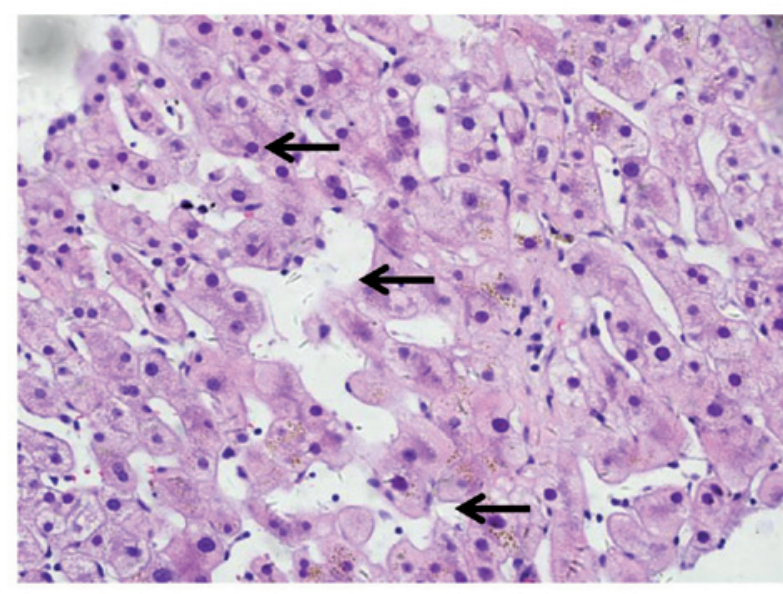

Figure 3. Pathological hematoxylin and eosin staining demonstrating distinctive sinusoidal dilatation (arrows) in the liver of a patient with gastric cancer who received oxaliplatin-containing chemotherapy (magnification, x400).

was demonstrated in the portal-venous phase (Fig. 2). In addition, the patient received liver biopsy. Tissue sections (5- $\mu \mathrm{m}$ thick) were fixed in formalin at room temperature, and paraffin-embedded liver samples were stained at room temperature with hematoxylin and eosin for standard histological examination using a light microscope. Pathological examination indicated distinctive multifocal sinusoidal dilatation without piecemeal necrosis, hepatic rosette formation and emperipolesis (Fig. 3). Written informed consent was obtained from the patient prior to publication of the present case report.

\section{Discussion}

HSOS, also known as veno-occlusive disease, is an obliterative venulitis of the terminal hepatic venules (6). It occurs as a result of cytoreductive therapy prior to hematopoietic stem cell transplantation (HSCT), following oxaliplatin-containing chemotherapy for colorectal carcinoma metastatic to the liver, in patients taking pyrrolizidine alkaloid-containing herbal remedies $(5,13,14)$. It is histologically characterized by sinusoidal dilatation, hepatocyte necrosis and obliterated hepatic venules due to damage to the sinusoidal endothelial cells (4). Sinusoidal changes induced by chemotherapeutic agents or pyrrolizidine alkaloid-containing herbals are caused by a direct toxicity to sinusoidal endothelial cells (12). In addition, toxic agents have an adverse effect on bone marrow progenitors of endothelial cells that are responsible for the repair of sinusoidal lesions (15). The clinical presentations of HSOS include jaundice, right upper-quadrant pain and tender hepatomegaly, ascites and unexplained weight gain. The diagnosis of a HSOS result from HSCT or pyrrolizidine alkaloid (PA)-containing herbals is based on a history of taking PAs-containing herbs and a classical triad of weight gain, painful hepatomegaly and jaundice $(13,14)$. However, clinical manifestations of the patients with oxaliplatin-induced HSOS appear to be mild or absent (10). Thus, it is challenge to define HSOS in patients who have received oxaliplatin-contained chemotherapy. Notably, patients with oxaliplatin-induced HSOS may give rise to elevated morbidity rates and increased bleeding risk. Thus, it is important to identify HSOS in patients who receive oxaliplatin-contained chemotherapy.

The diagnosis of HSOS is established by histological examination; however, the patchy nature of HSOS incurs sampling variability that confines the diagnostic yield of the biopsy. Previous studies have demonstrated that CT and/or MRI scans may provide excellent strategies for the screening of oxaliplatin-induced $\operatorname{HSOS}(8,10,11,16)$. In the present study, heterogeneous and predominantly hypoattenuation of the hepatic parenchyma was a distinctive radiological sign of oxaliplatin-induced HSOS on the CT scan. In addition, reticular hypointensity was an important indicator of oxaliplatin-induced HSOS observed from MRI in the patient. These imaging signs were also reported in previous studies $(8,10,11,16)$. Notably, various studies have demonstrated that oxaliplatin-induced HSOS manifested as focal liver lesions and mimicked metastatic colon cancer in the liver $(17,18)$. Thus, HSOS should have been differentiated from metastatic gastric cancer. Details of history, careful observation of radiological findings, and histological examination provide assistance in distinguishing oxaliplatin-induced HSOS from metastatic cancer.

In conclusion, the present case report detailed oxaliplatin-induced HSOS in a patient with gastric cancer. HSOS should be considered as one of the potential causes of newly-developed hepatic lesions in patients with gastric cancer who receive oxaliplatin. The present case highlights that oxaliplatin may induce HSOS not only in metastatic colon cancer in the liver, but also in other types of cancer, such as gastric cancer. Further studies are required to identify characteristics that predispose individuals to HSOS secondary to oxaliplatin and the prognosis of oxaliplatin-induced HSOS in gastric cancer.

\section{Acknowledgments}

The present study was supported by the National NaturalScience Foundation of China (grant nos. 81570555 and 81270506).

\section{References}

1. De Manzoni G, Marrelli D, Baiocchi GL, Morgagni P, Saragoni L, Degiuli M, Donini A, Fumagalli U, Mazzei MA, Pacelli F, et al: The italian research group for gastric cancer (GIRCG) guidelines for gastric cancer staging and treatment: 2015. Gastric Cancer 20: 20-30, 2017.

2. Sabanathan D, Eslick GD and Shannon J: Use of neoadjuvant chemotherapy plus molecular targeted therapy in colorectal liver metastases: A Systematic review and meta-analysis. Clin Colorectal Cancer 15: e141-e147, 2016.

3. Robinson SM, Mann J, Vasilaki A, Mathers J, Burt AD, Oakley F, White SA and Mann DA: Pathogenesis of FOLFOX induced sinusoidal obstruction syndrome in a murine chemotherapy model. J Hepatol 59: 318-326, 2013.

4. Valla DC and Cazals-Hatem D: Sinusoidal obstruction syndrome. Clin Res Hepatol Gastroenterol 40: 378-385, 2016.

5. Fan CQ and Crawford JM: Sinusoidal obstruction syndrome (hepatic veno-occlusive disease). J Clin Exp Hepatol 4: 332-346, 2014.

6. Plessier A, Rautou PE and Valla DC: Management of hepatic vascular diseases. J Hepatol 56 (Suppl 1): S25-S38, 2012.

7. Rubbia-Brandt L, Audard V, Sartoretti P, Roth AD, Brezault C, Le Charpentier M, Dousset B, Morel P, Soubrane O, Chaussade $\mathrm{S}$, et al: Severe hepatic sinusoidal obstruction associated with oxaliplatin-based chemotherapy in patients with metastatic colorectal cancer. Ann Oncol 15: 460-466, 2004. 
8. Han NY, Park BJ, Kim MJ, Sung DJ and Cho SB: Hepatic parenchymal heterogeneity on contrast-enhanced ct scans following oxaliplatin-based chemotherapy: Natural history and association with clinical evidence of sinusoidal obstruction syndrome. Radiology 276: 766-774, 2015.

9. Han NY, Park BJ, Sung DJ, Kim MJ, Cho SB, Lee CH, Jang YJ, Kim SY, Kim DS, Um SH, et al: Chemotherapy-induced focal hepatopathy in patients with gastrointestinal malignancy: Gadoxetic acid-enhanced and diffusion-weighted MR imaging with clinical-pathologic correlation. Radiology 271: 416-425, 2014.

10. Shin NY, Kim MJ, Lim JS, Park MS, Chung YE, Choi JY, Kim KW and Park YN: Accuracy of gadoxetic acid-enhanced magnetic resonance imaging for the diagnosis of sinusoidal obstruction syndrome in patients with chemotherapy-treated colorectal liver metastases. Eur Radiol 22: 864-871, 2012.

11. Ward J, Guthrie JA, Sheridan MB, Boyes S, Smith JT, Wilson D, Wyatt JI, Treanor D and Robinson PJ: Sinusoidal obstructive syndrome diagnosed with superparamagnetic iron oxide-enhanced magnetic resonance imaging in patients with chemotherapy-treated colorectal liver metastases. J Clin Oncol 26: 4304-4310, 2008.

12. Anurathapan U, Pakakasama S, Mekjaruskul P, Sirachainan N, Songdej D, Chuansumrit A, Charoenkwan P, Jetsrisuparb A, Sanpakit K, Pongtanakul B, et al: Outcomes of thalassemia patients undergoing hematopoietic stem cell transplantation by using a standard myeloablative versus a novel reduced-toxicity conditioning regimen according to a new risk stratification. Biol Blood Marrow Transplant 20: 2066-2071, 2014.
13. Li X, Yang X, Xu D, Li Q, Kong X, Lu Z, Bai T, Xu K, Ye J and Song Y: Magnetic resonance imaging findings in patients with pyrrolizidine alkaloid-induced hepatic sinusoidal obstruction syndrome. Clin Gastroenterol Hepatol 15: 955-957, 2017.

14. Kan X, Ye J, Rong X, Lu Z, Li X, Wang Y, Yang L, Xu K, Song Y and Hou X: Diagnostic performance of contrast-enhanced CT in pyrrolizidine alkaloids-induced hepatic sinusoidal obstructive syndrome. Sci Rep 6: 37998, 2016.

15. DeLeve LD: Liver sinusoidal endothelial cells and liver regeneration. J Clin Invest 123: 1861-1866, 2013.

16. O'Rourke TR, Welsh FK, Tekkis PP, Lyle N, Mustajab A, John TG, Peppercorn D and Rees M: Accuracy of liver-specific magnetic resonance imaging as a predictor of chemotherapy-associated hepatic cellular injury prior to liver resection. Eur J Surg Oncol 35: 1085-1091, 2009.

17. Choi JH, Won YW, Kim HS, Oh YH, Lim S and Kim HJ: Oxaliplatin-induced sinusoidal obstruction syndrome mimicking metastatic colon cancer in the liver. Oncol Lett 11: 2861-2864, 2016.

18. Arakawa Y, Shimada M, Utsunomya T, Imura S, Morine Y, Ikemoto T, Hanaoka J, Sugimoto $\mathrm{K}$ and Bando $\mathrm{Y}$ : Oxaliplatin-related sinusoidal obstruction syndrome mimicking metastatic liver tumors. Hepatol Res 43: 685-689, 2013. 